\title{
Probabilistic Models for Gene Silencing Data
}

Florian Markowetz

Dezember 2005

Dissertation zur Erlangung des Grades

eines Doktors der Naturwissenschaften (Dr. rer. nat.)

am Fachbereich Mathematik und Informatik

der Freien Universität Berlin 
1. Referent: Prof. Dr. Martin Vingron

2. Referent: Prof. Dr. Klaus-Robert Müller

Tag der Promotion: 26. April 2006 


\section{Preface}

Acknowledgements This work was carried out in the Computational Diagnostics group of the Department of Computational Molecular Biology at the Max Planck Institute for Molecular Genetics in Berlin. I thank all past and present colleagues for the good working atmosphere and the scientific - and sometimes maybe not so scientific-discussions.

Especially, I am grateful to my supervisor Rainer Spang for suggesting the topic, his scientific support, and the opportunity to write this thesis under his guidance. I thank Michael Boutros for providing the expression data and for introducing me to the world of RNAi when I visited his lab at the DKFZ in Heidelberg. I thank Anja von Heydebreck, Jörg Schultz, and Martin Vingron for their advice and counsel as members of my $\mathrm{PhD}$ commitee.

During the time I worked on this thesis, I enjoyed fruitful discussions with many people. In particular, I gratefully acknowledge Jacques Bloch, Steffen Grossmann, Achim Tresch, and Chen-Hsiang Yeang for their contributions. Special thanks go to Viola Gesellchen, Britta Koch, Stefanie Scheid, Stefan Bentink, Stefan Haas, Dennis Kostka, and Stefan Röpcke, who read drafts of this thesis and greatly improved it by their comments.

Publications Parts of this thesis have been published before. Chapter 2 grew out of lectures I gave in 2005 at the Instiute for Theoretical Physics and Mathematics (IPM) in Tehran, Iran, and at the German Conference on Bioinformatics (GCB), Hamburg, Germany. I thank Prof. Mehrdad Shahshahani (Tehran) and Prof. Stefan Kurtz (Hamburg) for inviting me. Chapter 3 gathers results of two conference papers: the first one at the Workshop on Distributed Statistical Computing (DSC 2003) in Vienna, Austria [82], and the second one at the Conference on Artifical Intelligence and Statistics (AISTATS), in Barbados, 2005 [81. Parts of chapter 4 were previously published in the journal Bioinformatics [80].

Figures This thesis reproduces three figures from other publications. I thank Prof. Danny Reinberg and Prof. Jules Hoffmann for the friendly permissions to reproduce Fig. 1.1 and Fig. 1.2, respectively. Fig. 1.3 is reproduced with permission from www. ambion. com.

Florian Markowetz

Berlin, December 2005 


\section{Contents}

Preface

1 Introduction 1

1.1 Signal transduction and gene regulation . . . . . . . . . . . . 1

1.2 Gene silencing by RNA interference . . . . . . . . . . . . . . 4

1.3 Thesis organization . . . . . . . . . . . . . 7

2 Statistical models of cellular networks 9

2.1 Conditional independence models . . . . . . . . . . . . . . . 9

2.2 Bayesian networks . . . . . . . . . . . . . . . . . 14

2.3 Score based structure learning . . . . . . . . . . . . . . . 17

2.4 Benchmarking . . . . . . . . . . . . . . . . . . . 24 24

2.5 A roadmap to network reconstruction . . . . . . . . . . . 25

3 Inferring transcriptional regulatory networks 27

3.1 Graphical models for interventional data . . . . . . . . . . . . . . 27

3.2 Ideal interventions and mechanism changes . . . . . . . . . . . . . . 29

3.3 Pushing interventions at single nodes . . . . . . . . . . . . . 32

3.4 Pushing in conditional Gaussian networks . . . . . . . . . 36

4 Inferring signal transduction pathways 45

4.1 Non-transcriptional modules in signaling pathways . . . . . . . . . . 45

4.2 Gene silencing with transcriptional phenotypes . . . . . . . . . . . 50

4.3 Accuracy and sample size requirements . . . . . . . . . . . . . 58

4.4 Application to Drosophila immune response . . . . . . . . . . . . 60

5 Summary and outlook 65

Bibliography 69

Notation and Definitions 83

Zusammenfassung

Curriculum Vitae 87 